
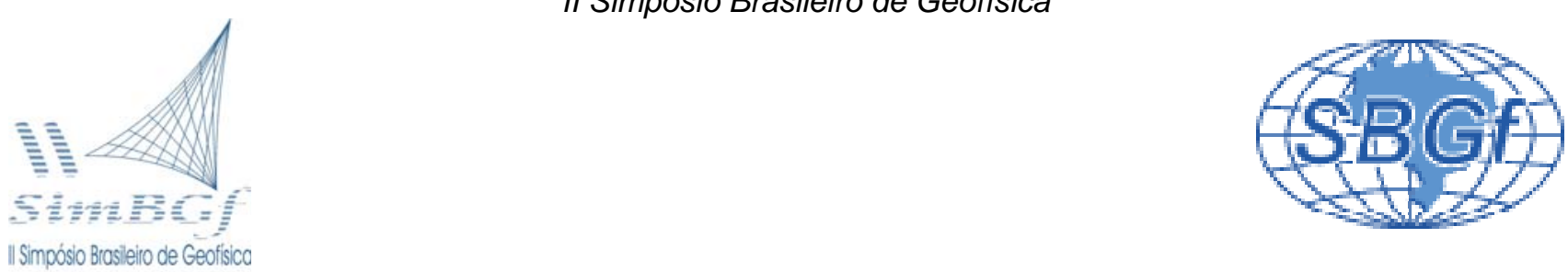

21 a 23 de Setembro de 2006 , Natal - RN

\title{
Gradiente e Fluxo Geotérmico no Estado do Paraná
}

Antonio Jorge de Lima Gomes (ajlgomes@on.br) e Valiya M. Hamza (hamza@on.br), Observatório Nacional, RJ, Brasil.

Copyright 2006, SBGf - Sociedade Brasileira de Geofísica

Este texto foi preparado para a apresentação no II Simpósio de Geofísica da Sociedade Brasileira de Geofísica, Natal, 21-23 de setembro de 2006. Seu conteúdo foi revisado pela Comissão Tecno-científica do II SR-SBGf mas não necessariamente representa a opinião da SBGf ou de seus associados. E proibida a reprodução total ou parcial deste material para propósitos comerciais sem prévia autorização da SBGf.

\section{Resumo}

Apresenta-se neste trabalho resultados de uma análise integrada dos dados geotérmicos do Estado do Paraná, realizados em 2006. Os trabalhos foram realizados pelo Laboratório de Geotermia do Observatório Nacional como parte integrante de um projeto de Avaliação de Recursos Geotermais da Bacia do Paraná. Os novos dados adquiridos no Estado do Paraná em conjunto com os já existentes, desde a década de 1970, permitiram caracterizações geotérmicas dos segmentos regionais principais. A compilação atual engloba medidas experimentais num total de 54 localidades, distribuídas em 43 municípios. A maior parte do Estado apresentou gradientes térmicos na faixa de 22 a $32^{\circ} \mathrm{C} / \mathrm{km}$ e fluxo geotérmico entre 40 e $100 \mathrm{~mW} / \mathrm{m}^{2}$. Verificam-se valores de fluxo geotérmico inferiores a $50 \mathrm{~mW} / \mathrm{m}^{2}$ na região costeira, o que é característico de regiões précambrianas. A região de Arco de Ponta Grossa apresentou valores de fluxo geotérmico na faixa de 50 a $60 \mathrm{~mW} / \mathrm{m}^{2}$, indicando que os vestígios térmicos das atividades magmáticas do período Cretáceo são praticamente desprezíveis. Por outro lado, constatamos valores relativamente elevados de gradiente e do fluxo geotérmico nas regiões Centro-Norte e Centro-Sul do Estado. Essas regiões possuem potencial para recursos geotermais de baixa entalpia $e$ as características geológicas e geotérmicas também favorecem possíveis ocorrências de maturação de hidrocarbonetos nas camadas abaixo da formação Serra Geral.

\section{Introdução}

Grande parte do Estado do Paraná situa-se na Bacia do Paraná. Desde a década de 1970 têm sido efetuadas diversas investigações geotérmicas na área da Bacia do Paraná e também nas suas áreas vizinhas. Destacamos os trabalhos de Meister (1973), Vitorello et al (1978), Araújo (1978), Vitorello et al (1980), Eston et al (1983), Santos (1987), Hurter (1986), Hurter e Hamza (1987), Del Rey e Hamza (1989), Hurter e Pollack (1996) e Del Rey (1989). Posteriormente Hamza e Muñoz (1996), Hurter e Pollack (1996), Gomes e Hamza (2004 e 2005) e Hamza et al (2005), apresentaram compilações de dados geotérmicos da Bacia do Paraná e das áreas vizinhas. Neste trabalho, nosso objetivo é mapear o campo térmico da área compreendida pelo Estado do Paraná. A compilação atual engloba medidas experimentais num total de 54 localidades, distribuídas em 43 municípios. Deste total, quarenta e seis dados já faziam parte das diversas publicações citadas anteriormente, enquanto que os oito restantes são novos, adquiridos como parte deste trabalho. Utilizamos também dados de fontes termais de Hurter et al $(1987,1983)$ com geotermômetros de SiO2, Na/k e Na-K-Ca. O mapeamento do gradiente e do fluxo geotérmico permitiu a avaliação das variações no campo térmico na área de estudo. Os locais dos dados são apresentados na Figura (1).

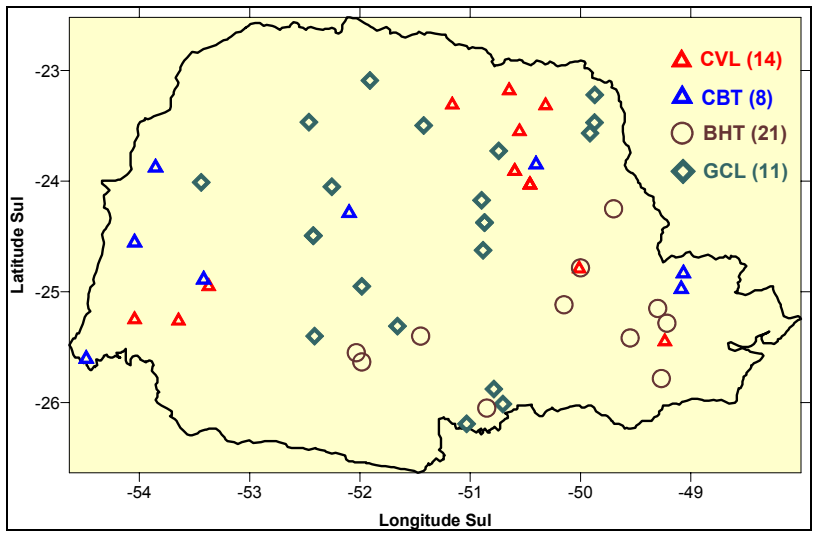

Figura 1 - Locais dos dados geotérmicos no Estado do Paraná. As siglas CVL, BHT, CBT e GCL referem-se aos métodos utilizados.

\section{Metodologia de Gradiente Térmico}

Foram utilizados quatro métodos distintos para determinar os gradientes térmicos, designados de convencional (CVL), temperatura do fundo de poço de petróleo (BHT), temperatura estável do fundo de poço (CBT) e o método geoquímico (GCL). As características principais dos métodos utilizados e estudos comparativos de confiabilidade foram apresentadas por Gomes (2003) e Hamza et al (2005).

O método convencional (CVL) é utilizado para determinar o gradiente em intervalos de profundidades selecionados, com base em resultados de perfilagem térmica. As correções efetuadas incluem efeitos de mudanças climáticas e de topografia local. Nos poços rasos com indícios de perturbações térmicas foram aplicadas correções para minimizar os efeitos de mudanças climáticas e de topografia local. Para um matriz de coeficientes $A_{M x N}$, com $M>N$, e para um vetor $\hat{y}$ de dados observados o critério de mínimos quadrados fornece estimativas que satisfazem este modelo. O método de 
mínimos quadrados permite estimativas dos coeficientes de ajuste linear. A incerteza na determinação de profundidade é pequena em relação à de temperatura. Desta forma a profundidade foi considerada como variável independente e a temperatura como variável dependente. No presente caso, para um conjunto de $\mathrm{N}$ pares de dados $\left(\mathrm{z}_{\mathrm{i}}, \mathrm{T}_{\mathrm{i}}\right)$ os coeficientes são o gradiente térmico $(\Gamma)$ e o intercepto $\left(T_{0}\right)$.

O método de temperatura do fundo de poço de petróleo (BHT) é utilizado geralmente para determinação de gradiente e fluxo geotérmico em poços de petróleo. A incerteza neste método é oriunda principalmente de uso de termômetros de baixa exatidão para as medidas. Utilizam-se as temperaturas do fundo do poço e da média anual da superfície, que ajustadas em profundidade permitem a determinação do Gradiente. A relação utilizada para a determinação do gradiente térmico é:

$$
\Gamma=\left(T_{F P}-T_{0}\right) / H=\sum_{i=1}^{N}(d T / d z)_{i} h_{i} / \sum h_{i}
$$

Onde: $T_{F P}$ é a temperatura do fundo do poço, $T_{0}$ a temperatura média anual da superfície, $\mathrm{H}$ a profundidade do poço, $N$ o número de camadas e $h$ a espessura da camada. As medições das temperaturas BHT, efetuadas em poços de petróleo, precisam ser corrigidas dos efeitos perturbadores das atividades de perfuração. As medidas múltiplas de temperaturas, foram corrigidas pelos métodos propostos por Lachenbruch e Brewer (1959) e Middleton (1980). Devido a características operacionais de aquisição, os dados precisam ser corrigidos dos efeitos perturbadores das atividades de perfuração. Nos casos em que havia apenas uma medida de temperatura utilizou-se a relação empírica da AAPG (1976). Os gradientes calculados pelo método BHT são médias de gradientes intervalares, que mudam conforme as variações nas condutividades térmicas das camadas interceptadas pelo poço.

O método de temperatura estável de fundo do poço (CBT) foi utilizado em casos em que o campo térmico do poço é alterado pelo fluxo de fluidos no seu interior. O princípio do método é baseado no fato que a perturbação térmica é praticamente nula na parte inferior do poço. As medições de temperaturas são geralmente efetuadas utilizando termômetros de precisão e, portanto, não necessitam de correções. Por outro lado, o grau de incerteza deste método é maior, já que a falta de informações apropriadas dificultam a determinação exata da temperatura média anual da superfície nos locais de poços. O gradiente pelo CBT é determinado pela seguin te relação:

$$
T_{C B T}=T_{0}+q \sum_{i=1}^{N} R_{i} h_{i}
$$

onde $R_{i}$ é a resistividade térmica da camada $i$. O termo do somatório se refere à resistência térmica cumulativa das formações presentes até o fundo do poço onde foi efetuada a medida de temperatura.

No método geoquímico (GCL) as temperaturas dos reservatórios são estimadas através de resultados dos com geotermômetros de Sílica $\left(\mathrm{SiO}_{2}\right)$, Sódio-Potássio $(\mathrm{Na}-\mathrm{K})$, e Sódio-Potássio-Cálcio (Na-K-Ca), estas em profundidade, sendo que foram calculadas, respectivamente, pelas seguintes relações:

a) Fournier (tipo SPV, 1981):

$$
T_{P}=[1309(5,19-\log S)-273,15]
$$

b) Fournier (tipo PMV, 1981):

$$
T_{P}=[1522(5,75-\log S)-273,15]
$$

c) Verma (1995):

$$
T_{P}=C_{1}+C_{2} S+C_{3} S^{2}+C_{5} S^{3}+C_{5} \log S
$$

Onde: $S$ é a concentração de sílica em ppm e $C_{1}$ a $C_{5}$ são coeficientes numéricos. $O$ calculo do gradiente por este método utiliza a seguinte relação:

$$
\Gamma_{G C L}=\left(T_{p}-T_{S}\right) /(m . K)
$$

Onde $T_{P}$ é a temperatura do reservatório, $T_{S}$ é a temperatura média anual da superfície e $m$ uma constante igual a $680 \pm 67{ }^{\circ} \mathrm{Cm}^{2} W^{1}$. Na determinação dos gradientes o modelo de Verma (1995) foi adotado para os valores finais, pela sua qualidade estatística.

\section{Estimativas de Gradiente Térmico por local}

Os resultados permitiram determinações de gradientes em 54 localidades, distribuídas por 43 municípios do Estado. Estes são apresentados nas Tabelas (1), (2), (3) e (4), , respectivamente, pelos métodos CVL, CBT, BHT e GCL.

Tabela (1) - Gradiente Térmico pelo método CVL.

\begin{tabular}{|c|c|c|c|c|}
\hline \multirow{2}{*}{ Município } & \multicolumn{2}{|c|}{ Coordenadas } & \multicolumn{2}{c|}{$\boldsymbol{\Gamma}\left({ }^{\circ} \mathbf{C} / \mathbf{k m}\right)$} \\
\cline { 2 - 5 } & Long. & Lat. & Médio & $\sigma$ \\
\hline Cascavel & $-53,3717$ & $-24,9511$ & 35,9 & 1,1 \\
\hline Curitiba & $-49,2353$ & $-25,4491$ & 18,3 & 0,1 \\
\hline Castro & $-50,0119$ & $-24,7911$ & 10,9 & 1,6 \\
\hline Congoinhas & $-50,5536$ & $-23,5511$ & 30,9 & 0,3 \\
\hline C. Procópio & $-50,6467$ & $-23,1811$ & 30,8 & 1,0 \\
\hline Curiúva (AA11-PR) & $-50,4583$ & $-24,0325$ & 25,5 & 1,7 \\
\hline Curiúva (CA-3-PR) & $-50,4582$ & $-24,0324$ & 19,7 & 0,6 \\
\hline Curiúva (Z-13-PR) & $-50,4583$ & $-24,0325$ & 25,3 & 0,9 \\
\hline Lindoeste & $-53,6449$ & $-25,2616$ & 24,1 & 1,0 \\
\hline Londrina & $-51,1628$ & $-23,3103$ & 30,2 & 1,0 \\
\hline Medianeira & $-54,0431$ & $-25,2500$ & 33,0 & 0,5 \\
\hline P. Grossa & $-50,3167$ & $-23,3167$ & 33,5 & 0,3 \\
\hline Sapopema (SPP1) & $-50,5969$ & $-23,9108$ & 26,1 & 1,4 \\
\hline Sapopema (SPP2) & $-50,5969$ & $-23,9108$ & 39,0 & 0,8 \\
\hline
\end{tabular}

Tabela (2) - Gradiente Térmico pelo método CBT.

\begin{tabular}{|c|c|l|c|c|}
\hline \multirow{2}{*}{ Município } & \multicolumn{2}{|c|}{ Coordenadas } & \multicolumn{2}{c|}{$\left.\Gamma{ }^{\circ} \mathbf{C} / \mathbf{k m}\right)$} \\
\cline { 2 - 5 } & Long. & Lat. & Médio & $\sigma$ \\
\hline Altonia & $-53,8536$ & $-23,8778$ & 20,5 & 3,1 \\
\hline Cachoeirinha & $-49,0667$ & $-24,8333$ & 14,9 & 2,2 \\
\hline Cascavel & $-53,4173$ & $-24,8891$ & 27,5 & 3,2 \\
\hline Figueira & $-50,4031$ & $-23,8492$ & 18,4 & 1,0 \\
\hline Foz do Iguaçu & $-54,4818$ & $-25,6057$ & 26,1 & 3,2 \\
\hline Iretama & $-52,0982$ & $-24,2876$ & 23,0 & 3,9 \\
\hline Mal. C. Rondon & $-54,0431$ & $-24,5541$ & 21,7 & 3,9 \\
\hline Tunas do Paraná & $-49,0858$ & $-24,9744$ & 10,4 & 1,6 \\
\hline
\end{tabular}


Tabela (2) - Gradiente Térmico pelo método BHT.

\begin{tabular}{|c|c|c|c|c|}
\hline \multirow{2}{*}{ Município } & \multicolumn{2}{|c|}{ Coordenadas } & \multicolumn{2}{c|}{$\left.\Gamma \mathbf{(}^{\circ} \mathbf{C} / \mathbf{k m}\right)$} \\
\cline { 2 - 5 } & Long. & Lat. & Médio & $\sigma$ \\
\hline Alto Piquiri & $-53,4400$ & $-24,0103$ & 26,1 & 3,9 \\
\hline Ângulo & $-51,9100$ & $-23,0900$ & 32,6 & 3,9 \\
\hline Apucarana & $-51,4214$ & $-23,4969$ & 20,3 & 2,4 \\
\hline Campo Mourão & $-52,2544$ & $-24,0511$ & 22,9 & 3,4 \\
\hline C. Abreu (CA-1-PR) & $-52,4239$ & $-24,4928$ & 13,4 & 2,0 \\
\hline C. Abreu (CA-2-PR) & $-52,4238$ & $-24,4927$ & 23,0 & 3,5 \\
\hline Chapéu do Sol & $-51,9833$ & $-24,9500$ & 18,5 & 2,8 \\
\hline Guarapuava & $-51,6600$ & $-25,3100$ & 28,3 & 4,2 \\
\hline Jacarezinho & $-49,8708$ & $-23,2208$ & 26,3 & 3,9 \\
\hline J. Távora & $-49,8722$ & $-23,4717$ & 18,6 & 2,8 \\
\hline L. do Sul & $-52,4111$ & $-25,4008$ & 25,5 & 3,8 \\
\hline Mallet & $-50,7861$ & $-25,8778$ & 28,1 & 4,2 \\
\hline Monjolinho (Mo1PR) & $-50,8706$ & $-24,3744$ & 20,8 & 3,1 \\
\hline Monjolinho (Mo2PR) & $-50,8706$ & $-24,3744$ & 20,8 & 3,1 \\
\hline Ortigueira & $-50,8964$ & $-24,1733$ & 27,4 & 4,1 \\
\hline Quatiguá & $-49,9136$ & $-23,5667$ & 19,3 & 0,3 \\
\hline Reserva & $-50,8833$ & $-24,6250$ & 20,1 & 3,0 \\
\hline R. C. do Sul & $-50,7044$ & $-26,0133$ & 26,4 & 4,0 \\
\hline Rio Ivaí & $-52,4633$ & $-23,4664$ & 25,9 & 3,9 \\
\hline S. J. Serra & $-50,7411$ & $-23,7275$ & 26,5 & 4,0 \\
\hline U. Vitória & $-51,0333$ & $-26,1917$ & 25,0 & 3,8 \\
\hline
\end{tabular}

Tabela (4) - Gradiente Térmico pelo método GCL.

\begin{tabular}{|c|c|c|c|c|}
\hline \multirow{2}{*}{ Município } & \multicolumn{2}{|c|}{ Coordenadas } & \multicolumn{2}{c|}{$\left.\Gamma \mathbf{(}^{\circ} \mathbf{C} / \mathbf{k m}\right)$} \\
\cline { 2 - 5 } & Long. & Lat. & Médio & $\sigma$ \\
\hline A.Tamandaré & $-49,3000$ & $-25,1500$ & 13,3 & 2,0 \\
\hline Campo Largo & $-49,5500$ & $-25,4167$ & 17,5 & 2,6 \\
\hline Candói & $-52,0333$ & $-25,5500$ & 37,3 & 5,6 \\
\hline Candói (S. Clara) & $-51,9833$ & $-25,6333$ & 34,7 & 5,2 \\
\hline Castro & $-50,0000$ & $-24,7833$ & 22,7 & 3,4 \\
\hline Cerro Azul & $-49,2667$ & $-25,7833$ & 15,7 & 2,4 \\
\hline Colombo & $-49,2167$ & $-25,2833$ & 10,7 & 1,6 \\
\hline Guarapuava & $-51,4500$ & $-25,4000$ & 42,8 & 6,4 \\
\hline Jaguariaíva & $-49,7000$ & $-24,2500$ & 31,0 & 4,7 \\
\hline P. de Frontin & $-50,8500$ & $-26,0500$ & 52,8 & 7,9 \\
\hline Ponta Grossa & $-50,1500$ & $-25,1167$ & 29,0 & 4,4 \\
\hline
\end{tabular}

A maior parte do Estado do Paraná apresentou gradientes térmicos na faixa de 22 a $32^{\circ} \mathrm{C} / \mathrm{km}$. Os valores médios dos gradientes térmicos obtidos por método foram os seguintes: método CVL com resultados de 27,4 $\pm 0,9^{\circ} \mathrm{C} / \mathrm{km}$; para o método $\mathrm{BHT}$ tivemos $23,6 \pm 1,9$ ${ }^{\circ} \mathrm{C} / \mathrm{km}$; com o método CBT os dados apontaram 20,3 \pm $3,2^{\circ} \mathrm{C} / \mathrm{km}$ e pelo método $\mathrm{GCL}$ os valores constatados foram de $21,8 \pm 3,7^{\circ} \mathrm{C} / \mathrm{km}$.

Com base nos resultados das Tabelas (1) a (4) foram calculados valores médios representativos dos gradientes térmicos das formações geológicas, cujas idades variam de Arqueano ao Cretáceo. Apresenta-se na Figura (2) uma síntese da variação do gradiente no Estado por idade geológica.

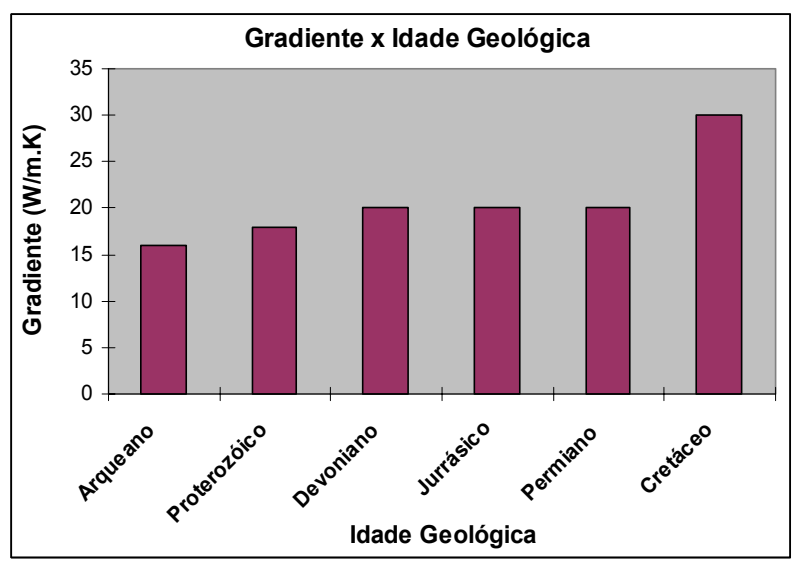

Figura 2 - Gradientes térmicos médios das formações no Estado do Paraná, classificadas por idade geológica.

\section{Determinação e resultados de Fluxo Geotérmico}

O fluxo geotérmico (q) quando aplicado num meio isotrópico (Kappelmeyer et al, 1974) é determinado pela lei de Fourier, que nos apresenta a seguinte relação:

$$
q=-k \cdot \frac{d T}{d x}
$$

Onde: $k$ é a condutividade média da formação e $d T / d X$ o gradiente térmico.

No método CVL a média harmônica de condutividade térmica é multiplicada pelo gradiente térmico do intervalo selecionado, para obter o valor do fluxo geotérmico (q):

$$
q=\Gamma \cdot \lambda_{m} \pm \sigma_{q}
$$

Onde: $\Gamma$ é o gradiente térmico, $\lambda_{m}$ a condutividade média e $\sigma_{q}$ o desvio padrão.

No método CBT o valor do fluxo geotérmico é dado pela relação:

$$
q=\left(T_{C B T}-T_{0}\right) / \sum_{i=1}^{N} R_{i} h_{i}
$$

Onde: $\mathrm{T}_{\mathrm{CBT}}$ é a temperatura estável do fundo do poço, $\mathrm{T}_{0}$ a de superfície, $R$ a resistência térmica e $h$ a espessura da camada em questão. A equação anterior pode ser escrita da seguinte forma:

$$
q=\left\{\frac{\left(T_{C B T}-T_{0}\right)}{\left(Z_{C B T}-Z_{0}\right)}\right\}\left\{\left(Z_{C B T}-Z_{0}\right) /\left(\sum_{i=1}^{N} R_{i} h_{i}\right)\right\}
$$

$\mathrm{Na}$ equação anterior o primeiro termo do lado direito representa o gradiente térmico aparente das camadas que se encontram no intervalo $\left(Z_{\mathrm{CBT}}-Z_{0}\right)$. Este gradiente é freqüentemente designado de "gradiente BHT".

O método de termometria geoquímica ( $G C L$ ) utiliza-se a relação empírica proposta por Swanberg e Morgan (1985): Este método é utilizado para estimar a magnitude 
do fluxo geotérmico em locais de surgências de fontes termo-minerais. Esta relação é a seguinte:

$$
T q_{z}=m \cdot q+T_{0}
$$

Onde: $T_{Q Z}$ representa temperatura da sílica, $T_{0}$ a temperatura média anual da superfície e $m$ uma constante cujo valor é $680^{\circ} \mathrm{Cm}^{2} / \mathrm{W}$.

Utilizamos nas avaliações de condutividade térmica resultados obtidos por Eston et al (1981), Hurter e Hamza (1987), Del Rey e Hamza (1989), Hurter e Pollack (1996) e Hamza et al (2005). Os resultados de fluxo geotérmico obtidos no presente trabalho pelos métodos (CVL), (BHT), (CBT) e (AQT) são apresentados, respectivamente, nas Tabelas (4), (5), (6) e (7), por município. Nessas tabelas $\sigma$ representa o desvio padrão.

Tabela (4) - Condutividade Térmica e Fluxo Térmico pelo método CVL.

\begin{tabular}{|c|c|c|c|c|}
\hline \multirow{2}{*}{ Município } & \multicolumn{2}{|c|}{$\boldsymbol{\lambda}\left(\mathbf{W} / \mathbf{m}^{*} \mathbf{K}\right)$} & \multicolumn{2}{c|}{$\mathbf{q}\left(\mathbf{m W} / \mathbf{m}^{\mathbf{2}}\right)$} \\
\cline { 2 - 5 } & Média & $\sigma$ & Médio & $\sigma$ \\
\hline Cascavel & 2,2 & 0,4 & 79 & 14 \\
\hline Curitiba & 2,7 & 0,5 & 49 & 09 \\
\hline Castro & 2,7 & 0,5 & 29 & 07 \\
\hline Congoinhas & 2,1 & 0,4 & 65 & 12 \\
\hline C. Procópio & 2,1 & 0,4 & 65 & 12 \\
\hline Curiúva (AA-11-PR) & 2,7 & 0,5 & 69 & 13 \\
\hline Curiúva (CA-3-PR) & 2,7 & 0,5 & 53 & 10 \\
\hline Curiúva (Z-13-PR) & 2,7 & 0,5 & 68 & 13 \\
\hline Lindoeste & 2,1 & 0,4 & 51 & 09 \\
\hline Londrina & 2,7 & 0,5 & 82 & 15 \\
\hline Medianeira & 2,1 & 0,4 & 69 & 13 \\
\hline P. Grossa & 2,6 & 0,3 & 86 & 9 \\
\hline Sapopema (SPP1) & 2,4 & 0,4 & 63 & 12 \\
\hline Sapopema (SPP2) & 2,4 & 0,4 & 94 & 17 \\
\hline
\end{tabular}

Tabela (5) - Condutividade Térmica e Fluxo Térmico pelo método BHT.

\begin{tabular}{|c|c|c|c|c|}
\hline \multirow{2}{*}{ Município } & \multicolumn{2}{|c|}{$\boldsymbol{\lambda}\left(\mathbf{W} / \mathbf{m}^{\star} \mathbf{K}\right)$} & \multicolumn{2}{c|}{$\mathbf{q}\left(\mathbf{m W} / \mathbf{m}^{\mathbf{2}}\right)$} \\
\cline { 2 - 5 } & Média & $\sigma$ & Médio & $\sigma$ \\
\hline Alto Piquiri & 2,1 & 0,4 & 55 & 13 \\
\hline Ângulo & 1,8 & 0,3 & 58 & 12 \\
\hline Apucarana & 2,1 & 0,4 & 42 & 09 \\
\hline Campo Mourão & 2,0 & 0,4 & 46 & 11 \\
\hline C. Abreu (CA-1-PR) & 2,1 & 0,4 & 28 & 07 \\
\hline C. Abreu (CA-2-PR) & 2,1 & 0,4 & 48 & 11 \\
\hline Chapéu do Sol & 2,1 & 0,4 & 39 & 09 \\
\hline Guarapuava & 2,2 & 0,4 & 62 & 15 \\
\hline Jacarezinho & 2,6 & 0,5 & 69 & 16 \\
\hline J. Távora & 2,8 & 0,5 & 52 & 12 \\
\hline L. do Sul & 2,1 & 0,4 & 54 & 13 \\
\hline Mallet & 2,7 & 0,5 & 74 & 17 \\
\hline Monjolinho (Mo1PR) & 2,8 & 0,5 & 58 & 14 \\
\hline Monjolinho (Mo2PR) & 2,8 & 0,5 & 58 & 14 \\
\hline Ortigueira & 2,7 & 0,5 & 73 & 17 \\
\hline
\end{tabular}

\begin{tabular}{|c|c|c|c|c|}
\hline Quatiguá & 2,9 & 0,5 & 55 & 10 \\
\hline Reserva & 2,7 & 0,5 & 53 & 12 \\
\hline R. C. do Sul & 2,6 & 0,5 & 70 & 16 \\
\hline Rio Ivaí & 2,4 & 0,4 & 62 & 15 \\
\hline S. J. Serra & 2,4 & 0,4 & 64 & 15 \\
\hline U. Vitória & 2,6 & 0,5 & 65 & 15 \\
\hline
\end{tabular}

Tabela (6) - Condutividade Térmica e Fluxo Térmico pelo método CBT.

\begin{tabular}{|c|c|c|c|c|}
\hline \multirow{2}{*}{ Município } & \multicolumn{2}{|c|}{$\boldsymbol{\Lambda}\left(\mathbf{W} / \mathbf{m}^{*} \mathbf{K}\right)$} & \multicolumn{2}{c|}{$\mathbf{q}\left(\mathbf{m W} / \mathbf{m}^{\mathbf{2}}\right)$} \\
\cline { 2 - 5 } & Média & $\sigma$ & Médio & $\sigma$ \\
\hline Altonia & 2,9 & 0,5 & 60 & 14 \\
\hline Cachoeirinha & 2,9 & 0,5 & 43 & 10 \\
\hline Cascavel & 2,2 & 0,4 & 61 & 13 \\
\hline Figueira & 2,4 & 0,4 & 44 & 08 \\
\hline Foz do Iguaçu & 2,2 & 0,4 & 57 & 13 \\
\hline Iretama & 3,1 & 0,6 & 71 & 18 \\
\hline Mal. C. Rondon & 2,1 & 0,4 & 46 & 12 \\
\hline Tunas do Paraná & 2,4 & 0,4 & 25 & 06 \\
\hline
\end{tabular}

Tabela (7) - Condutividade Térmica e Fluxo Térmico pelo método GCL.

\begin{tabular}{|c|c|c|c|c|}
\hline \multirow{2}{*}{ Município } & \multicolumn{2}{|c|}{$\boldsymbol{\lambda ( \mathbf { W } / \mathbf { m } ^ { \star } \mathbf { K } )}$} & \multicolumn{2}{c|}{$\mathbf{q}\left(\mathbf{m W} / \mathbf{m}^{\mathbf{2}}\right)$} \\
\cline { 2 - 5 } & Média & $\sigma$ & Médio & $\sigma$ \\
\hline A.Tamandaré & 3,2 & 0,4 & 43 & 08 \\
\hline Campo Largo & 2,1 & 0,4 & 37 & 09 \\
\hline Candói & 2,7 & 0,5 & 101 & 24 \\
\hline Candói (S. Clara) & 2,7 & 0,5 & 94 & 22 \\
\hline Castro & 2,7 & 0,5 & 61 & 14 \\
\hline Cerro Azul & 2,7 & 0,5 & 42 & 10 \\
\hline Colombo & 2,7 & 0,5 & 29 & 07 \\
\hline Guarapuava & 1,6 & 0,3 & 68 & 16 \\
\hline Jaguariaíva & 2,2 & 0,4 & 68 & 16 \\
\hline P. de Frontin & 2,1 & 0,4 & 111 & 26 \\
\hline Ponta Grossa & 2,6 & 0,3 & 75 & 13 \\
\hline
\end{tabular}

Com base nos valores observados nas tabelas (4) a (8), constata-se que o Estado possui valores de fluxo geotérmico entre 40 e $100 \mathrm{~mW} / \mathrm{m}^{2}$. O menor fluxo está na região costeira cujos valores são inferiores a $50 \mathrm{~mW} / \mathrm{m}^{2}$. Observa-se uma região de Leste para Oeste na direção aproximada do Arco de Ponta grossa com fluxo médio de $60 \mathrm{~mW} / \mathrm{m}^{2}$. Duas regiões Centro-Sul e Centro-Norte, apresentam características semelhantes, ambas com fluxo maior que $65 \mathrm{~mW} / \mathrm{m}^{2}$.

\section{Mapeamento do Gradiente e Fluxo Geotérmico}

A melhor forma de examinar as variações regionais do gradiente geotérmico é através da elaboração de mapas. A distribuição do gradiente e do fluxo em escala regional no Estado foi realizada através do método de superfícies numéricas e os resultados de gradiente e fluxo são apresentados, respectivamente nas Figuras (3) e (4). Nota-se no mapa da figura (3) que a faixa costeira PréCambriana é caracterizada por valores de gradientes térmicos menores que $20^{\circ} \mathrm{C} / \mathrm{km}$. Na região do Arco de Ponta Grossa os gradientes térmicos estão na faixa de 
10 a $15^{\circ} \mathrm{C} / \mathrm{km}$. Por outro lado, os mapas revelam que as regiões centro-norte e centro-sul do Estado do Paraná são caracterizados por gradientes térmicos na faixa de 15 a $36^{\circ} \mathrm{C} / \mathrm{km}$. O mesmo padrão das variações também é observado na distribuição de fluxo geotérmico, ilustrada na Figura (4).

\section{Discussão e Conclusões}

As feições presentes nos mapas de gradiente e de fluxo geotérmico são consideradas como indicativo da forte influência das estruturas geológicas no campo termal da crosta superior no Estado do Paraná. Esta característica pode ser melhor compreendida considerando a seqüência da evolução tectônica das regiões principais: área costeira, Arco de Ponta Grossa, segmentos centronorte e centro-sul da Bacia do Paraná e o derrame basáltico da formação Serra Geral. A primeira região compreende os baixos valores de gradiente e fluxo térmico da região costeira (gradiente $<18^{\circ} \mathrm{C} / \mathrm{km}$ e fluxo $<50 \mathrm{~mW} / \mathrm{m}^{2}$ ), cujo resultado é considerado normal em áreas pré-cambrianas. A segunda região segue de Leste para Oeste, seguindo aproximadamente a direção do Arco de Ponta Grossa, originando uma aparente estabilização dos valores do gradiente na faixa de $22^{\circ} \mathrm{C} / \mathrm{km}$ e fluxo de $60 \mathrm{~mW} / \mathrm{m}^{2}$. A terceira e quarta regiões estão respectivamente situadas no Centro-Sul e no Centro-Norte, apesar de opostas, apresentam gradiente e fluxo maiores que $30^{\circ} \mathrm{C} / \mathrm{km}$ e $65 \mathrm{~mW} / \mathrm{m}^{2}$, sugerindo uma resposta do meio geológico na transferência de calor geotérmico por refração. As regiões com valores de fluxo $\left(>65 \mathrm{~mW} / \mathrm{m}^{2}\right.$ ) situadas na parte interna da Bacia do Paraná, dentro do Estado, apresentam potencialidade de exploração de recursos geotermais de baixa entalpia e favorável à potencialidade de maturação de hidrocarbonetos.

\section{Agradecimentos}

O primeiro autor deste trabalho é recipiente de bolsa de Doutorado pela Coordenadoria de Aperfeiçoamento de Ensino Superior - CAPES.

\section{Referências}

American Association of Petroleum Geologists (AAPG), 1976. Basic data file from AAPG Geothermal Survey of North America: Univ. of Oklahoma, Norman.

Araújo, R. L. C., 1978. Pesquisas de fluxo térmico na chaminé alcalina de Poços de Caldas, Tese de Mestrado, Universidade de São Paulo, São Paulo, Brasil.

Del Rey, A.C. and Hamza, V.M., 1989. Terrestrial Heat Flow variations in the northeastern parts of the state of São Paulo : A case for transport of geothermal heat by interfracture fluid flows, In Hydrogeological Regimes and their subsurface thermal effects, Geophysical Monograph, 47, American Geophysical Union, p. 137-148.

Eston, S.M., Hamza, V.M. e Becker, E.A., 1981, Pesquisas Geotérmicas na Exploração de Hidrocarbonetos na Bacia do Paraná, Relatório 16.379, Instituto de Pesquisas Tecnológicas do Estado de São Paulo-IPT.
Gomes, A.J.L., Hamza, V.M., 2003, Avaliação de Recursos Geotermais do Estado do Rio de Janeiro, $8^{\circ}$.international Congress of the Brazilian Geophysical Society, Rio de Janeiro.

Gomes, A.J.L. e Hamza, V.M., 2004. Mapeamento de Gradientes Geotérmicos no Estado de São Paulo, In: $1^{\circ}$ Simpósio Regional de Geofísica, Anais, Cd-rom, São Paulo, 26-28 de setembro, 4p.

Gomes, A.J.L. and HAMZA, V.M., 2005. Gradiente e Fluxo Geotérmico do Estado de Santa Catarina, $9^{\circ}$ International Congress of the Brazilian Geophysical Society, 6p., Cd-rom, Salvador, Brasil.

Hamza, V.M., Silva Dias, F.J.S., Gomes, A.J.L. and Terceros, Z.G.D., 2005. Numerical and Functional Representations of Regional Heat Flow in South America, Physics of the Earth and Planetary Interiors, Volume 152, 4, p.223-256.

Hamza, V.M. and Muñoz, M., 1996. Heat Flow map of South America, Geothermics, Vol. 25, no 6, pp. 599-646.

Hurter, S.J., Eston, S.M., Hamza, V.M., 1983. Coleção Brasileira de Dados Geotérmicos - Série 2: Fontes Termais. 1. ed. São Paulo: IPT publicação 1233.

Hurter, S.J., 1986. The use of chemical geothermometry and heat loss models in estimating terrestrial heat flow for low temperature hydrothermal systems. Rev. Bras. Geofisica 6, 33-42.

Hurter, S.J. e Hamza, V.M., 1987. Aplicação de Geotermômetros Químicos em águas de Fontes Brasileiras na determinação do Fluxo Geotérmico, Dissertação de Mestrado, IAG/USP, São Paulo.

Hurter, S.J. and Pollack, H.N., 1996. Terrestrial heat flow in the Paraná basin, Southern Brazil, J. Geophysics. Res., 101, p.8659-8672.

Lachenbruch, A.H. and Brewer, N., 1959. Dissipation of the temperature effect of drilling a well in Arctic Alaska, Bulletin, 1083-C, U.S. Geological Survey, Washington, USA, pp. 73-109.

Santos, J., Hamza, V.M., Shen, P.Y., 1986. A method for measurement of terrestrial heat flow density in water wells. Rev. Bras. Geofísica 4, 45-53.

Swanberg, C.A. and Morgan, P., 1985. Silica heat flow estimates and heat flow in the Colorado Plateau and adjacent areas. Journal of Geodynamics, 3; 65-85.

Verma, S.P., Santoyo, E., 1995. New improved equations for $\mathrm{Na} / \mathrm{K}$ and $\mathrm{SiO}_{2}$ Geothermometers by error propagation, World Geothermical Congress, Florence, Italy, pp. 963-968.

Vitorello, I., Hamza, V.M.; Pollack, H.N. and Araújo, R.L.C., 1978. Geothermal Investigations in Brazil, Rev. Brasil. Geociências, vol. 8, pp. 71-89.

Vitorello, I., Hamza, V.M. and Pollack, H.N., 1980. Terrestrial Heat Flow in the Brazilian Highlands, Journal of Geophysical Research, vol. 85, pp. 3778-3788. 


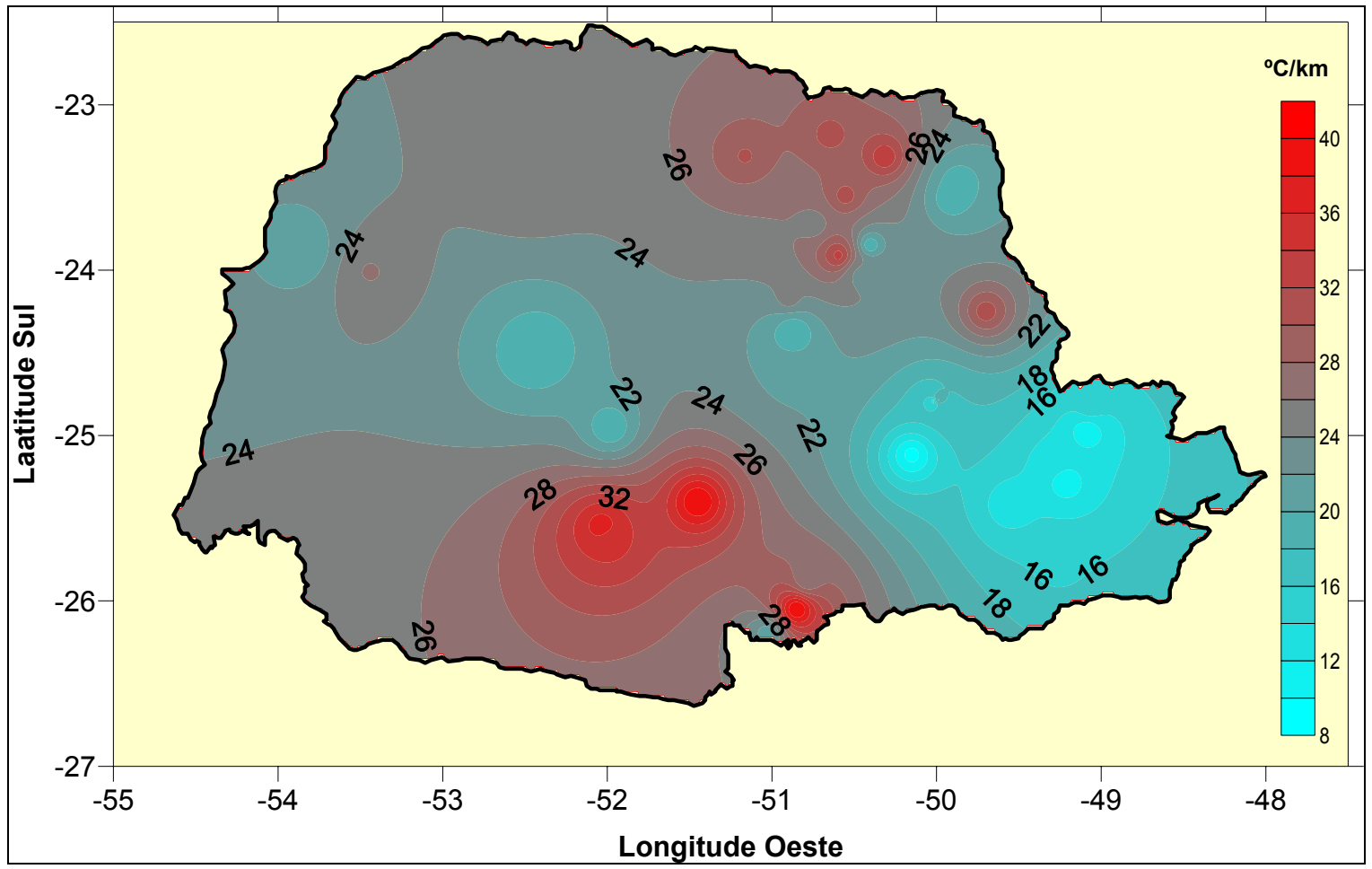

Figura 3 - Distribuição regional de gradiente geotérmico do Estado do Paraná.

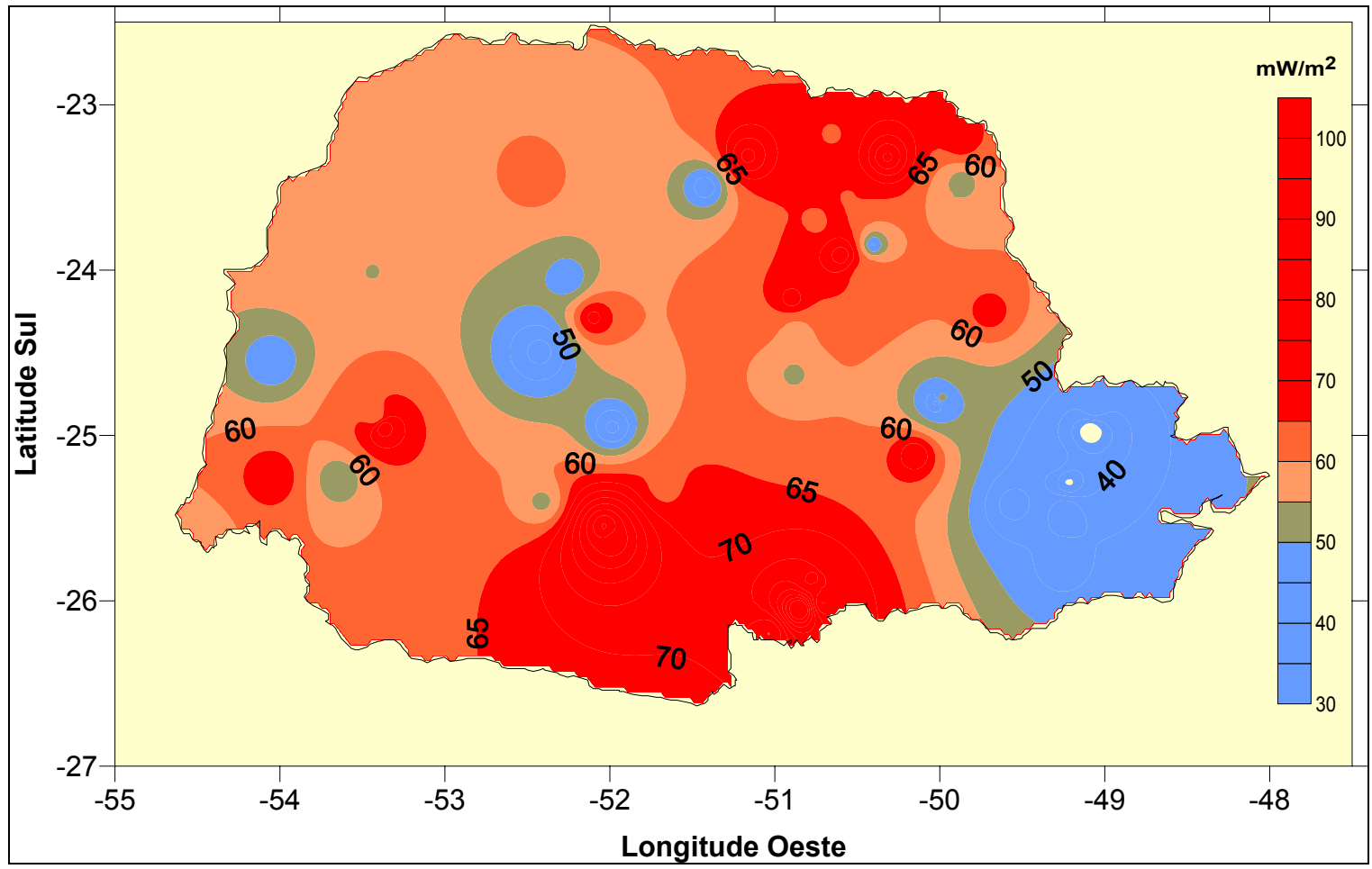

Figura 4 - Distribuição regional de fluxo geotérmico no Estado do Paraná. 